\title{
APPENDIX V. SHORE LABORATORY REPORT ON THE FORAMINIFERA FROM LEG 21 SITES, DEEP SEA DRILLING PROJECT ${ }^{1}$
}

\author{
Richard K. Olsson, Rutgers University, New Brunswick, New Jersey
}

Tables 1 through 24 show the distribution of planktonic foraminifera at Leg 21 sites, with indication of abundance and preservation. The symbols used in the tables are as follows:

Relative Abundance

$$
\begin{aligned}
& 0 \text { - Absent } \\
& 1 \text { - Rare (1-10 specimens) } \\
& 2 \text { - Moderately rare ( } 11-25 \text { specimens) } \\
& 3 \text { - Common (26-50 specimens) } \\
& 4 \text { - Abundant ( } 51-100 \text { specimens) } \\
& 5 \text { - Very abundant (100 specimens) }
\end{aligned}
$$

* - Hundred to thousands of specimens

P - Present

Preservation

$$
\begin{aligned}
& \text { D - Dissolution } \\
& \text { M - Mechanical erosion } \\
& \text { R - Reworked }
\end{aligned}
$$

\footnotetext{
${ }^{1}$ Editors note: This report was received after Volume 21 of the Initial Reports of the Deep Sea Drilling Project had gone to press.
}

TABLE 1

Distribution of Foraminifera, Hole 203

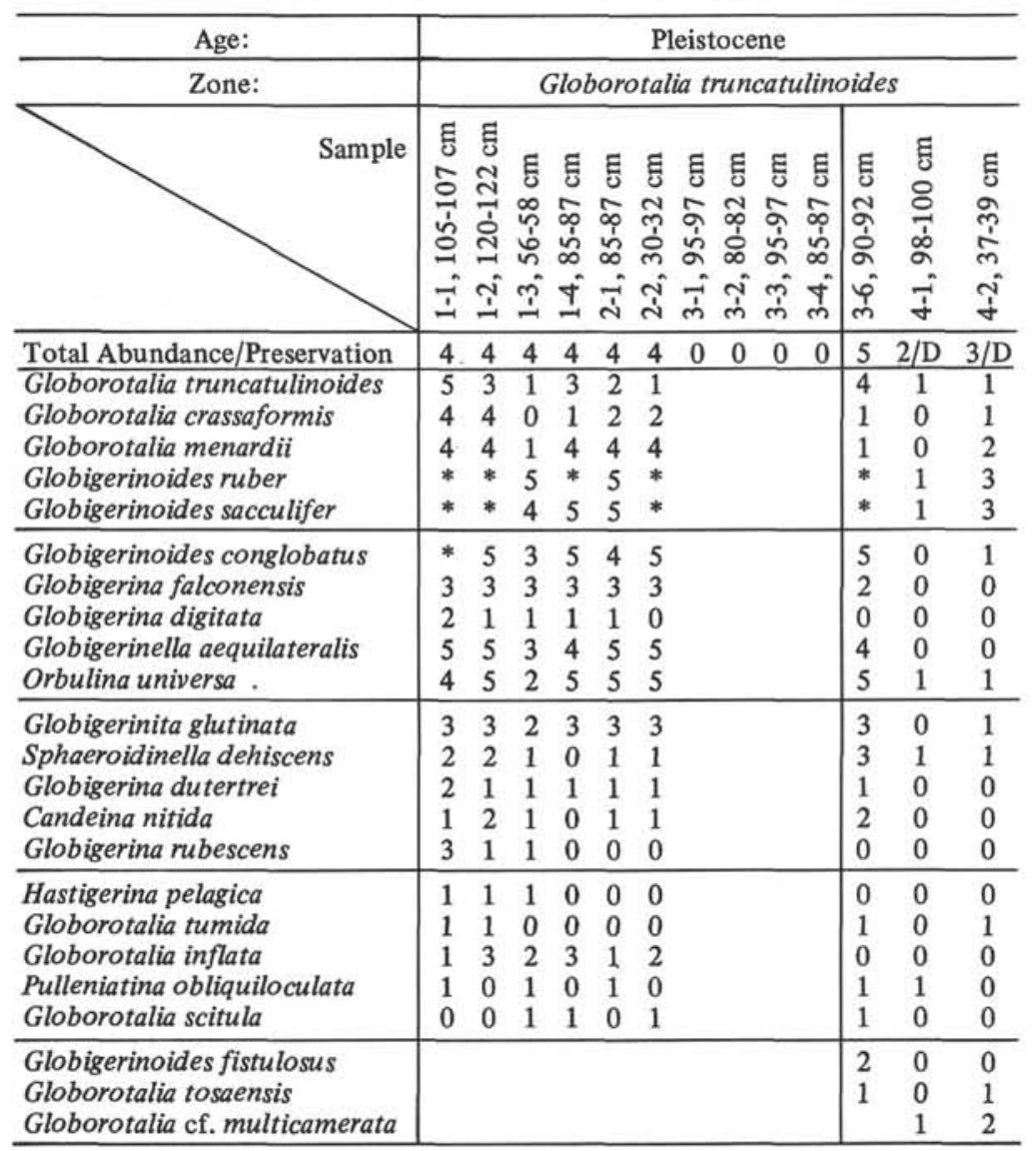


TABLE 2

Distribution of Foraminifera, Hole 205

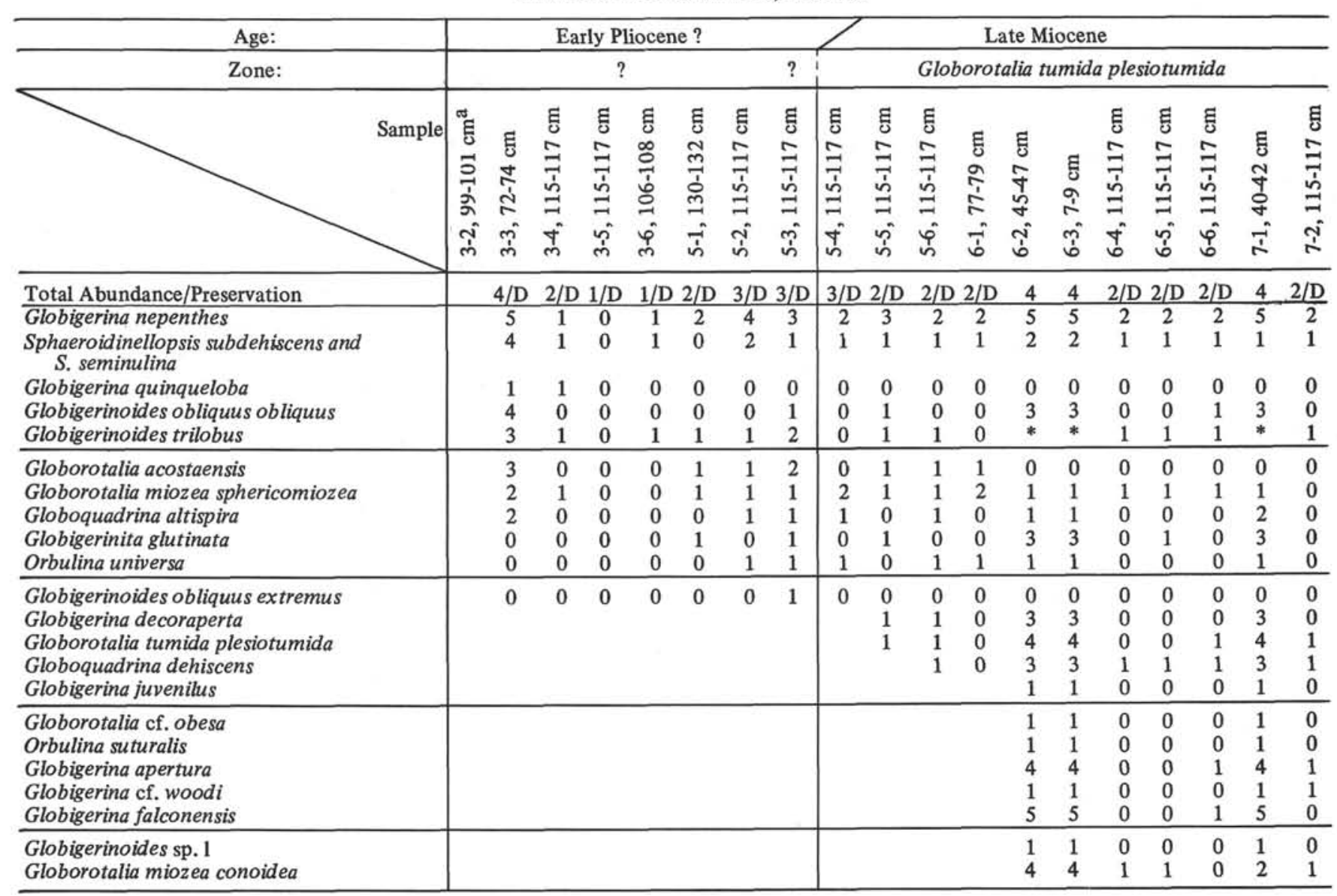

${ }^{\mathrm{a}}$ Barren. 
TABLE 3

Distribution of Foraminifera, Hole 205 - Continued

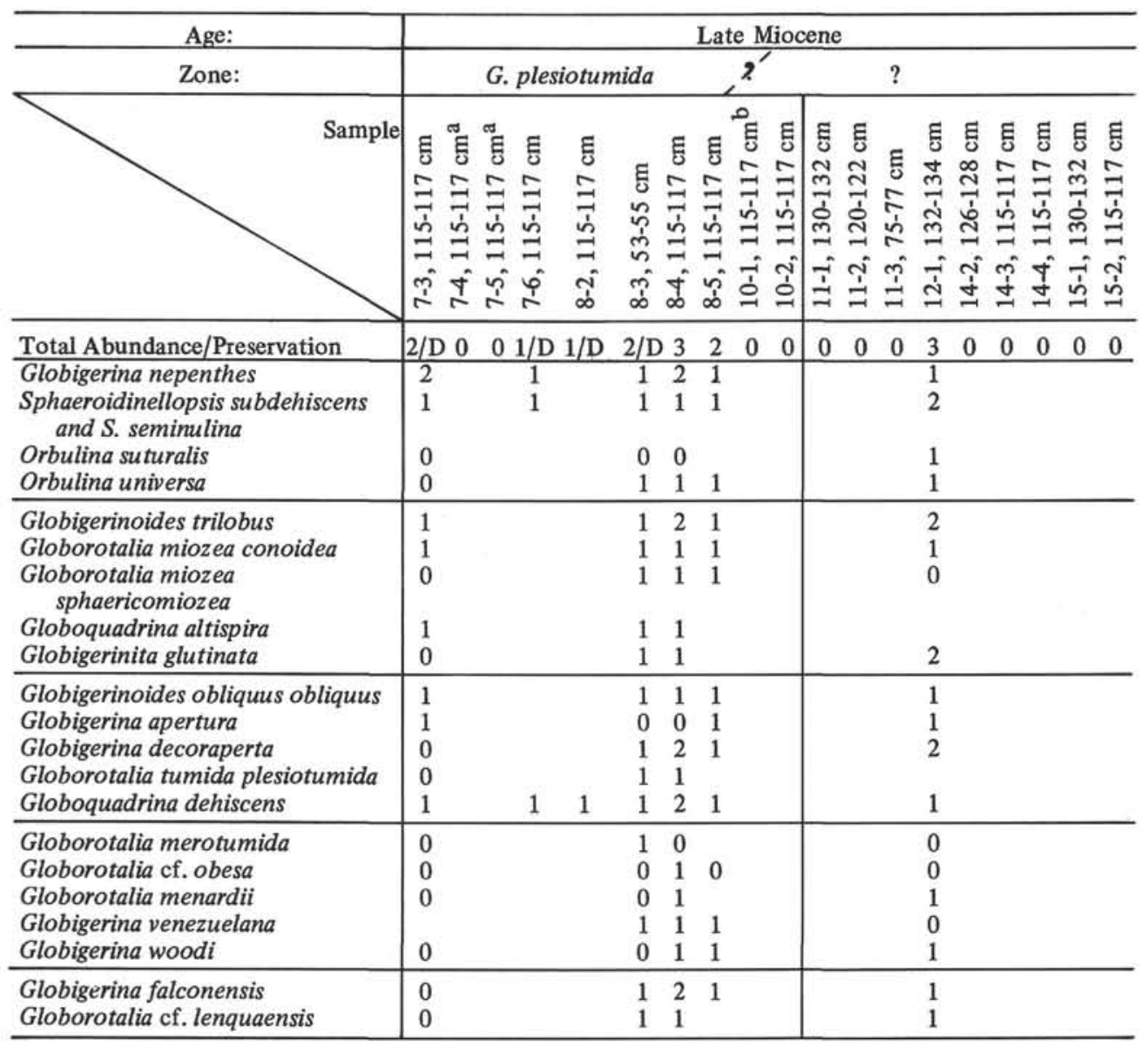

${ }^{\mathrm{a}}$ Barren, Volcanic ash.

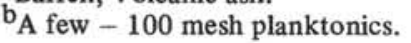

TABLE 4

Distribution of Foraminifera, Hole 205 - Continued

\begin{tabular}{|c|c|c|c|c|c|}
\hline Age: & \multicolumn{5}{|c|}{ Middle Miocene } \\
\hline Zone: & \multicolumn{5}{|c|}{ ? } \\
\hline Sample & 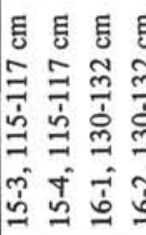 & 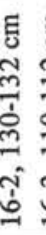 & 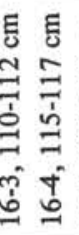 & 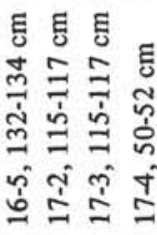 & 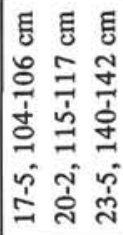 \\
\hline Total Abundance/Preservation & $\begin{array}{llll}0 & 0 & 2 & 2\end{array}$ & 2 & 22 & $\begin{array}{llll}3 & 0 & 0 & 0\end{array}$ & 220 \\
\hline $\begin{array}{l}\text { Sphaeroidinellopsis subdehiscens } \\
\quad \text { and S. seminulina } \\
\text { Globigerinoides trilobus } \\
\text { Globoquadrina altispira } \\
\text { Globigerinita glutinata } \\
\text { Orbulina universa }\end{array}$ & 11 & $\begin{array}{l}1 \\
1\end{array}$ & $\begin{array}{ll}1 & 1 \\
& 1 \\
1 & 1 \\
1 & 1\end{array}$ & $\begin{array}{l}2 \\
1 \\
1 \\
1\end{array}$ & $\begin{array}{ll}1 & 1 \\
1 & 2 \\
0 & 0 \\
0 & 1\end{array}$ \\
\hline $\begin{array}{l}\text { Globigerina decoraperta } \\
\text { Globoquadrina dehiscens } \\
\text { Orbulina suturalis } \\
\text { Globigerina apertura } \\
\text { Globigerina falconensis }\end{array}$ & $\begin{array}{ll}1 & \\
1 & 1 \\
1 & \end{array}$ & & $\begin{array}{ll}1 & 1 \\
1 & 1\end{array}$ & $\begin{array}{l}2 \\
1 \\
1\end{array}$ & $\begin{array}{ll}1 & 2 \\
1 & 1 \\
& \\
& 1\end{array}$ \\
\hline $\begin{array}{l}\text { Globigerinoides bollii } \\
\text { Globorotalia } \mathrm{cf} \text {. miozea miozeg } \\
\text { Globigerina praebulloides } \\
\text { Globorotalia } \mathrm{cf} \text {. praescitula } \\
\text { Globoquadrina larmeui }\end{array}$ & $\begin{array}{l}1 \\
1 \\
1\end{array}$ & & $\begin{array}{ll} & 1 \\
1 & 1 \\
1 & 1 \\
1 & 1\end{array}$ & $\begin{array}{l}1 \\
1 \\
1 \\
1\end{array}$ & $\begin{array}{r}1 \\
\\
1 \\
1 \\
1 \\
1 \\
1\end{array}$ \\
\hline
\end{tabular}


TABLE 5

Distribution of Foraminifera, Hole 206

\begin{tabular}{|c|c|c|c|c|c|c|c|c|c|c|c|c|c|c|c|c|c|c|}
\hline Age: & \multicolumn{18}{|c|}{ Pleistocene } \\
\hline Zone: & \multicolumn{18}{|c|}{ Globorotalia truncatulinoides } \\
\hline Sample & 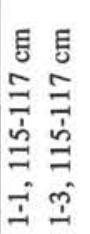 & 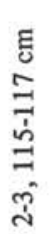 & 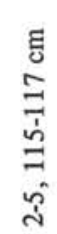 & 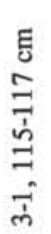 & 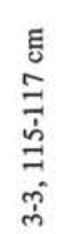 & 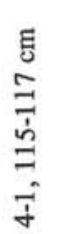 & 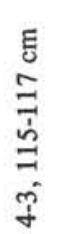 & 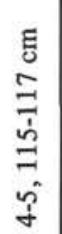 & $\begin{array}{l}E \\
\Xi \\
\exists \\
\ddot{n} \\
\vec{n}\end{array}$ & 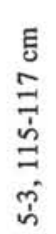 & 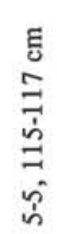 & 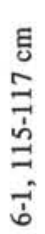 & 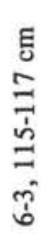 & 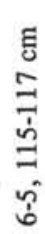 & & 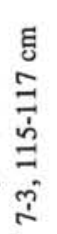 & 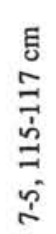 & $\begin{array}{l}E \\
0 \\
0 \\
5 \\
\dot{5} \\
\text { के }\end{array}$ \\
\hline Total Abundance/Preservation & $54 / D$ & 4/D & $4 / D$ & 4/D & 4/D & 4/D & $4 / D$ & 4/D & 5/D & 5/D & $5 / \mathrm{D}$ & 5 & 5 & 5 & & 4/D & 5/D & 5 \\
\hline Globorotalia truncatulinoides & 43 & 3 & 3 & 2 & 2 & 2 & 2 & 3 & 3 & 3 & 3 & 3 & 3 & 4 & 4 & 3 & 3 & 2 \\
\hline Globorotalia crassaformis & 53 & 3 & 3 & 3 & 4 & 3 & 3 & 3 & 4 & 4 & 4 & 5 & 4 & 4 & 4 & 4 & 4 & * \\
\hline Globorotalia inflata & $\begin{array}{ll}5 & 4\end{array}$ & 4 & 4 & 5 & 5 & 5 & 5 & 5 & * & * & * & * & * & * & * & 5 & * & * \\
\hline Globorotalia scitula & 31 & 1 & 1 & 1 & 1 & 1 & 1 & 1 & 1 & 1 & 1 & 1 & 1 & 2 & 2 & 1 & 1 & 1 \\
\hline Globorotalia crassula & 30 & 0 & 0 & 0 & 0 & 0 & 0 & 0 & 0 & 0 & 0 & 0 & 0 & 0 & 0 & 0 & 0 & 0 \\
\hline Globorotalia menardii & 10 & 0 & 0 & 1 & 1 & 1 & 1 & 1 & 2 & 1 & 1 & 2 & 2 & 1 & 1 & & 1 & 1 \\
\hline Globigerinoides ruber & 42 & 2 & 2 & 3 & 3 & 3 & 2 & 3 & 3 & 3 & 3 & 4 & 4 & 4 & 4 & 4 & 4 & 4 \\
\hline Globigerinoides trilobus & 31 & 1 & 1 & 2 & 2 & 2 & 2 & 2 & 2 & 2 & 2 & 2 & 2 & 3 & 3 & 3 & 3 & 3 \\
\hline Globigerina dutertrei & 21 & 1 & 2 & 2 & 2 & 2 & 2 & 2 & 2 & 2 & 2 & 2 & 2 & 0 & 1 & 1 & 1 & 1 \\
\hline Globigerinoides sacculifer & 10 & 0 & 0 & 0 & 0 & 0 & 0 & 0 & 0 & 0 & 1 & 1 & 0 & 1 & 1 & 1 & 1 & 1 \\
\hline Globigerina bulloides & 52 & 0 & 2 & 2 & 2 & 1 & 1 & 2 & 2 & 2 & 1 & 1 & 1 & 1 & 1 & 1 & 1 & 1 \\
\hline Globigerina falconensis & $\begin{array}{ll}5 & 4\end{array}$ & 3 & 4 & 5 & 5 & 5 & 5 & 5 & * & * & * & * & * & * & * & 5 & * & * \\
\hline Pulleniatina obliquiloculata & 10 & 2 & 0 & 0 & 1 & 1 & 1 & 1 & 2 & 3 & 3 & 4 & 4 & 4 & 3 & 3 & 2 & 1 \\
\hline Sphaeroidinella dehiscens & 10 & 1 & 0 & 0 & 0 & 0 & 1 & 0 & 1 & 1 & 2 & 2 & 2 & 3 & 3 & 1 & 1 & 1 \\
\hline Globigerinella aequilateralis & 31 & 1 & 1 & 0 & 2 & 1 & 1 & 1 & 1 & 1 & 1 & 1 & 1 & 2 & 2 & 1 & 1 & 1 \\
\hline Orbulina universa & 41 & 1 & 1 & 3 & 3 & 2 & 2 & 2 & 3 & 3 & 3 & 3 & 3 & 3 & 3 & 2 & 2 & 2 \\
\hline Globigerina pseudopachyderma & 33 & 4 & 4 & 5 & 5 & 5 & 5 & 5 & 5 & 5 & 5 & 5 & 4 & 4 & 4 & 4 & 4 & 4 \\
\hline Globerinoides conglobatus & & & 1 & 0 & 0 & 0 & 0 & 0 & 0 & 0 & 0 & 1 & 0 & 1 & 1 & 0 & 0 & 2 \\
\hline Globorotalia tumida & & & & & & & & & 2 & 1 & 0 & 2 & 2 & 3 & 3 & 1 & 1 & 2 \\
\hline Globoquadrina dehiscens & & & & & & & & & & & & & & $1 \mathbf{R}$ & 0 & 0 & 0 & $1 \mathrm{R}$ \\
\hline Globigerinita glutinata & & & & & & & & & & & & & & 2 & 2 & 2 & 2 & 2 \\
\hline
\end{tabular}


TABLE 6

Distribution of Foraminifera, Hole 206 - Continued

\begin{tabular}{|c|c|c|c|c|c|c|c|c|c|c|c|c|c|c|c|c|c|}
\hline Age: & \multicolumn{13}{|c|}{ Pleistocene } & \multicolumn{4}{|c|}{ Pliocene } \\
\hline \multirow[t]{2}{*}{ Zone: } & \multicolumn{13}{|c|}{ Globorotalia truncatulinoides } & \multicolumn{4}{|c|}{$\begin{array}{c}\text { Globorotalia } \\
\text { tosaensis }\end{array}$} \\
\hline & 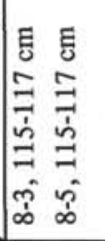 & 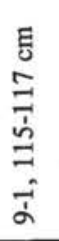 & 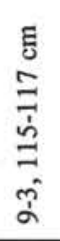 & 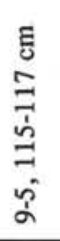 & 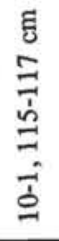 & 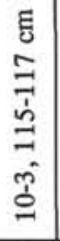 & 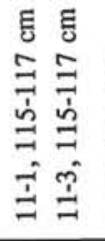 & 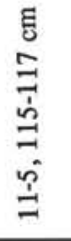 & $\begin{array}{l}E \\
\Xi \\
\bar{\Xi} \\
\dot{\Xi} \\
\vec{\Xi} \\
\vec{\Xi}\end{array}$ & 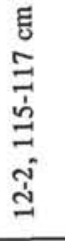 & 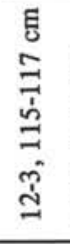 & 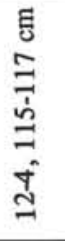 & 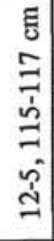 & 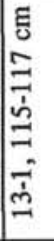 & 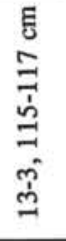 & $\begin{array}{l}E \\
\Xi \\
\Xi \\
\bar{\Xi} \\
\Xi \\
\exists \\
\dot{J}\end{array}$ & 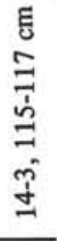 \\
\hline Total Abundance/Preservation & $54 / \mathrm{D}$ & $4 / \mathrm{D}$ & 5/D & 4/D & 4/D & $4 / \mathrm{D}$ & $54 / \mathrm{D}$ & 5/D & 4/D & 4/D & $5 / \mathrm{D}$ & $5 / \mathrm{D}$ & 5 & 5/D & 4/D & 4/D & 4/D \\
\hline Globorotalia truncatulinoides & $\begin{array}{ll}1 & 0\end{array}$ & 3 & 3 & 3 & 3 & 2 & 30 & 0 & 1 & 1 & 3 & 2 & 2 & 0 & 0 & 0 & 0 \\
\hline Globorotalia crassaformis & * 5 & 3 & 1 & 2 & 2 & 1 & $\begin{array}{ll}0 & 0\end{array}$ & 3 & 2 & 2 & 2 & 2 & 2 & 4 & 4 & 5 & 4 \\
\hline Globorotalia inflata & * 5 & 5 & 5 & 5 & 5 & 5 & * 5 & 4 & 5 & 5 & * & * & $*$ & * & 5 & 3 & 0 ? \\
\hline Globorotalia scitula & 10 & 1 & 1 & 1 & 1 & 1 & 21 & 1 & 1 & 1 & 1 & 1 & 1 & 2 & 1 & 1 & 1 \\
\hline Globorotalia crassula & $\begin{array}{ll}0 & 0 \\
\end{array}$ & 0 & 0 & 0 & 0 & 0 & $\begin{array}{ll}0 & 0 \\
\end{array}$ & 0 & 0 & 0 & 0 & 0 & 0 & 0 & 0 & 0 & 0 \\
\hline Globorotalia menardii & 10 & 0 & 0 & 0 & 0 & 0 & $\begin{array}{ll}0 & 0\end{array}$ & 0 & 0 & 0 & 0 & 0 & 0 & 0 & 0 & 0 & 0 \\
\hline Globigerinoides ruber & 44 & 3 & 4 & 4 & 4 & 3 & 40 & 5 & 4 & 3 & 4 & 4 & 4 & 4 & 4 & 3 & 3 \\
\hline Globigerinoides trilobus & 32 & 2 & 2 & 2 & 2 & 1 & 20 & 2 & 1 & 1 & 2 & 2 & 3 & 3 & 3 & 3 & 3 \\
\hline Globigerina dutertrei & 21 & 2 & 2 & 2 & 2 & 1 & 11 & 0 & 0 & 0 & 0 & 0 & 1 & 0 & 1 & 0 & 0 \\
\hline Globigerinoides sacculifer & $\begin{array}{ll}0 & 1 \\
\end{array}$ & 0 & 0 & 1 & 1 & 0 & 11 & 1 & 1 & 0 & 1 & 1 & 2 & 2 & 0 & 0 & 0 \\
\hline Globigerina bulloides & 10 & 2 & 2 & 1 & 1 & 1 & 11 & 1 & 0 & 1 & 1 & 1 & 1 & 1 & 1 & 0 & 0 \\
\hline Globigerina falconensis & & 5 & 5 & 5 & 5 & 5 & & 5 & 5 & 5 & * & * & $*$ & * & 5 & 4 & 4 \\
\hline Pulleniatina obliquiloculata & 12 & 5 & 5 & 1 & 2 & 2 & 10 & 0 & 0 & 0 & 0 & 0 & 0 & 0 & 0 & 0 & 0 \\
\hline Sphaeroidinella dehiscens & 11 & 1 & 2 & 1 & 1 & 1 & 20 & 2 & 1 & 0 & 0 & 0 & 1 & 1 & 1 & 0 & 0 \\
\hline Globigerinella aequilateralis & 21 & 1 & 1 & 1 & 1 & 0 & 20 & 0 & 0 & 0 & 1 & 1 & 2 & 2 & 2 & 1 & 0 \\
\hline Orbulina universa & 34 & 3 & 3 & 3 & 3 & 3 & 32 & 2 & 2 & 2 & 3 & 3 & 3 & 3 & 2 & 2 & 2 \\
\hline Globigerina pseudopachyderma & 33 & 3 & 3 & 3 & 3 & 3 & 53 & 4 & 4 & 4 & 5 & 5 & 4 & 4 & 4 & 4 & 4 \\
\hline Globigerinoides conglobatus & 12 & 2 & 1 & 0 & 0 & 0 & 10 & 3 & 0 & 0 & 1 & 1 & 1 & 1 & 1 & 0 & 0 \\
\hline Globorotalia tumida & 24 & 1 & 1 & 1 & 1 & 0 & 30 & $1 \mathrm{cf}$ & 0 & 0 & 0 & 0 & 2 & 2 & 2 & 1 & 1 \\
\hline Globoquadrina dehiscens & $\begin{array}{ll}0 & 0 \\
\end{array}$ & 0 & 0 & 0 & 0 & 0 & $\begin{array}{ll}0 & 0 \\
\end{array}$ & 0 & 0 & 0 & 0 & 0 & 0 & 0 & 0 & 0 & 0 \\
\hline Globigerinita glutinata & 33 & 3 & 3 & 3 & 3 & 3 & & 3 & 3 & 3 & 4 & 4 & 4 & 4 & 2 & 1 & 2 \\
\hline Globorotalia tosaensis & & & 1 & 1 & 1 & 1 & $\begin{array}{ll}0 & 0\end{array}$ & 0 & 0 & 1 & 2 & 2 & 2 & 3 & 3 & 2 & 1 \\
\hline Pulleniatina primalis & & & & 1 & 1 & 2 & 11 & 3 & 0 & 1 & 0 & 1 & 0 & 0 & 0 & 0 & 0 \\
\hline Globorotalia hirsuta & & & & & 1 & 2 & 23 & 3 & 0 & 0 & 0 & 0 & 0 & 0 & 0 & 0 & 0 \\
\hline Globorotalia cf. multicamerata & & & & & & & 10 & 0 & 1 & 1 & 0 & 1 & 1 & 1 & 1 & 1 & 1 \\
\hline Globorotalia acostaensis & & & & & & & & 2 & 0 & 0 & 0 & 0 & 0 & 0 & 0 & 0 & 0 \\
\hline Globorotalia numerosa & & & & & & & & & 1 & 0 & 0 & 0 & 0 & 1 & 0 & 0 & 0 \\
\hline Globigerinoides fistulosus & & & & & & & & & & & & & 1 & 0 & 0 & & 0 \\
\hline $\begin{array}{l}\text { Globigerinoides obliquus } \\
\text { Globorotalia praehirsuta }\end{array}$ & & & & & & & & & & & & & & & 1 & 0 & $\begin{array}{l}0 \\
4\end{array}$ \\
\hline Globorotalia pseudomiocenica & & & & & & & & & & & & & & & & & 2 \\
\hline
\end{tabular}


TABLE 7

Distribution of Foraminifera, Hole 206 - Continued

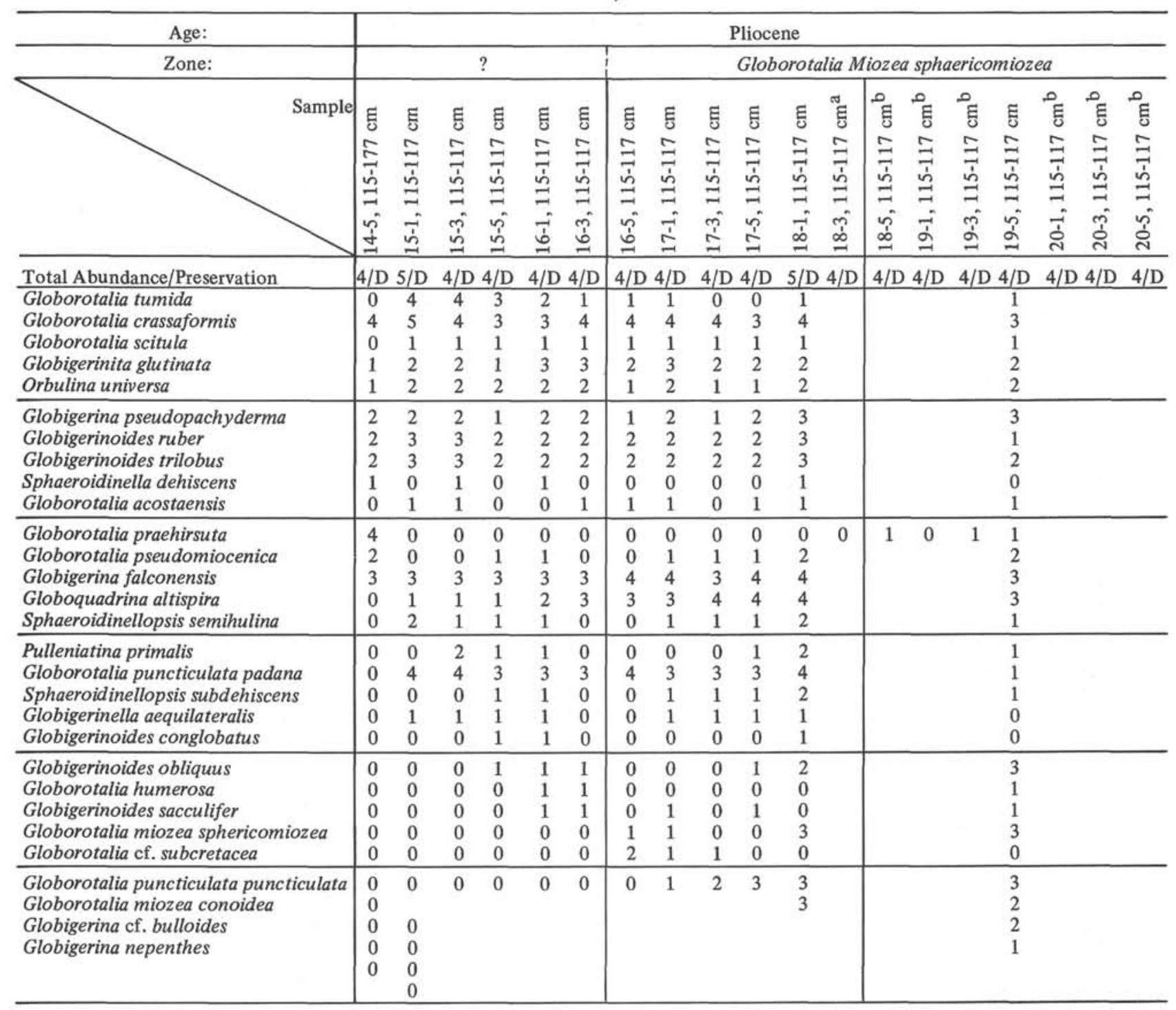

${ }^{\mathrm{a}}$ Chalky preservation.

${ }^{b}$ Chalky matrix. 
TABLE 8

Distribution of Foraminifera, Hole 206 - Continued

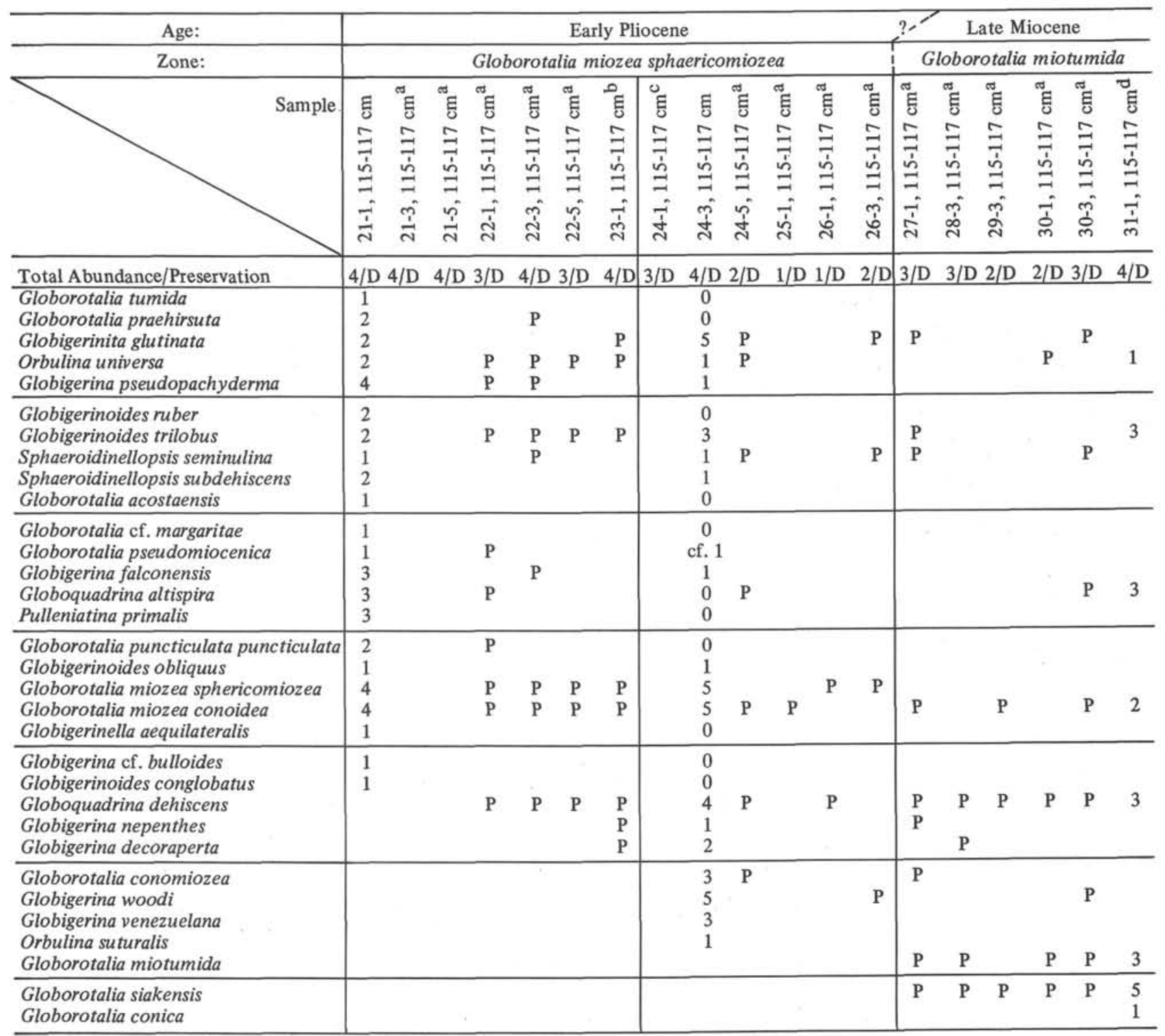

${ }^{a}$ Chalky matrix.

bome Recent or Pleistocene contamination.

${ }^{c}$ Recent or Pleistocene mix with chalky matrix.

$\mathrm{d}_{\text {Also some Recent or Pleistocene contamination. }}$ 
TABLE 9

Distribution of Foraminifera, Hole 206 - Continued

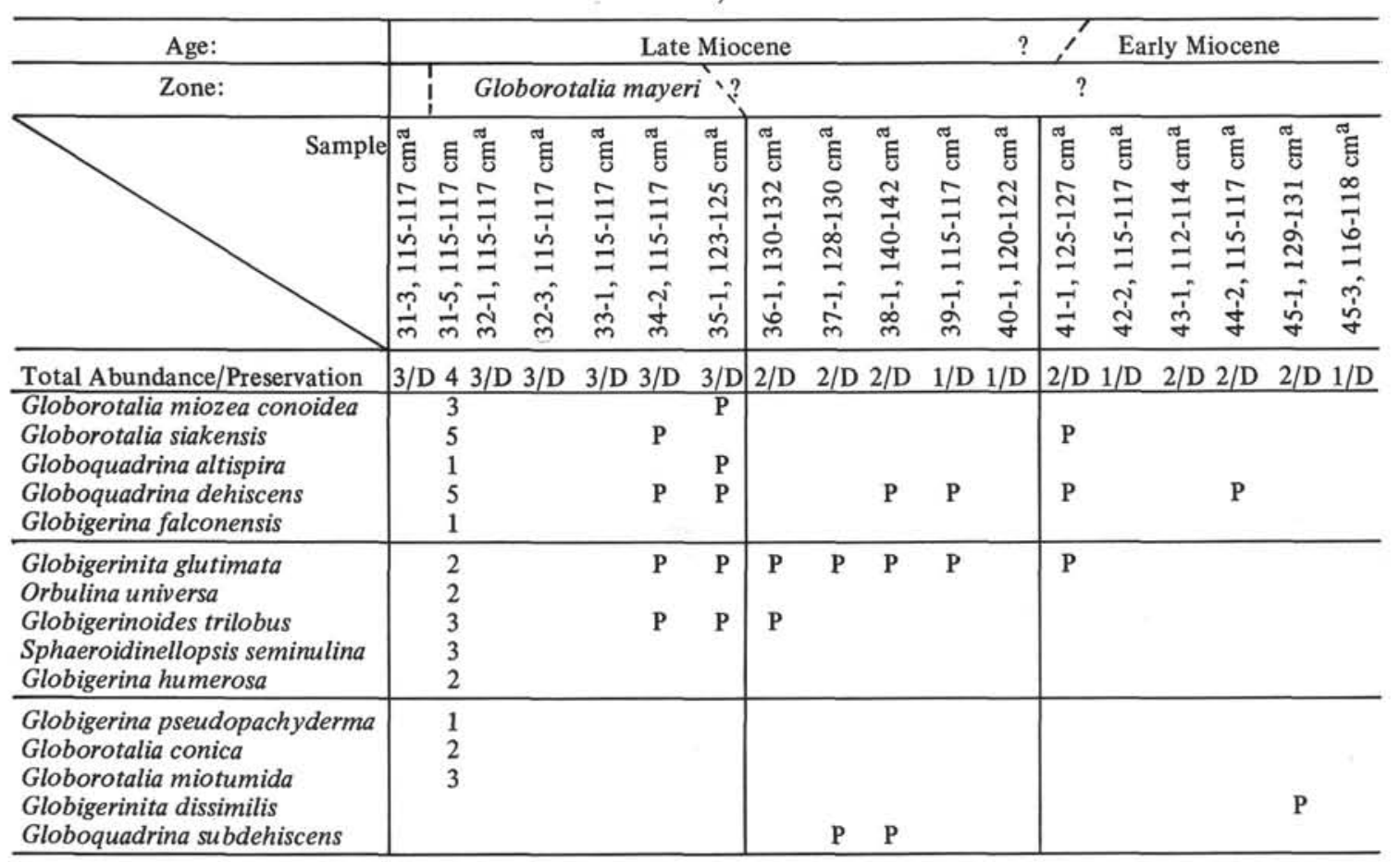

${ }^{\mathrm{a} C h a l k y ~ m a t r i x}$

TABLE 10

Distribution of Foraminifera, Hole 206C

\begin{tabular}{|c|c|c|c|c|c|c|c|c|c|c|c|c|}
\hline Age: & \multicolumn{7}{|c|}{$?$} & \multicolumn{5}{|c|}{ Oligocene ? } \\
\hline Zone: & & & & & & & ? & & & & & \\
\hline Sample & 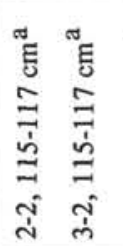 & 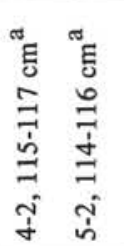 & 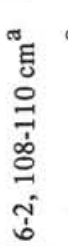 & 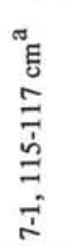 & 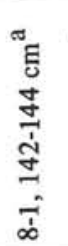 & 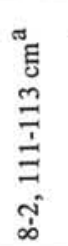 & 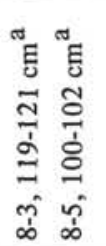 & 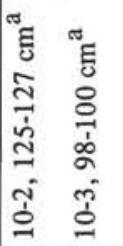 & 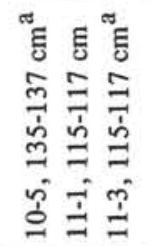 & 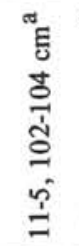 & 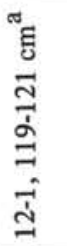 & 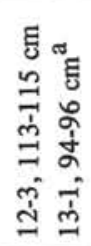 \\
\hline Total Abundance/Preservation & 1/D $1 / \mathrm{D}$ & $1 / \mathrm{D} 1 / \mathrm{D}$ & $1 / \mathrm{D}$ & $1 / \mathrm{D}$ & $1 / \mathrm{D}$ & $1 / \mathrm{D}$ & $01 / \mathrm{D}$ & $1 / \mathrm{D} 2 / \mathrm{D}$ & $1 / \mathrm{D} 01 / \mathrm{D}$ & 1/D & $1 / \mathrm{D}$ & $03 / \mathrm{D}$ \\
\hline $\begin{array}{l}\text { Globigerinids too poorly } \\
\quad \text { preserved to identify } \\
\text { Globigerinita dissimilis } \\
\text { Globigerinita pera } \\
\text { Globigerina unicava }\end{array}$ & P P & P P & $\mathrm{P}$ & $\mathrm{P}$ & $\mathrm{P}$ & $\mathrm{P}$ & $\mathrm{P}$ & P P & $\mathrm{P} \quad \mathrm{P}$ & $\mathrm{P}$ & $\mathrm{P}$ & $\begin{array}{l}\mathbf{P} \\
\mathbf{P} \\
\mathbf{P} \\
\mathbf{P}\end{array}$ \\
\hline
\end{tabular}

${ }^{\mathrm{a}}$ Chalky matrix. 
TABLE 11

Distribution of Foraminifera, Hole 206C - Continued

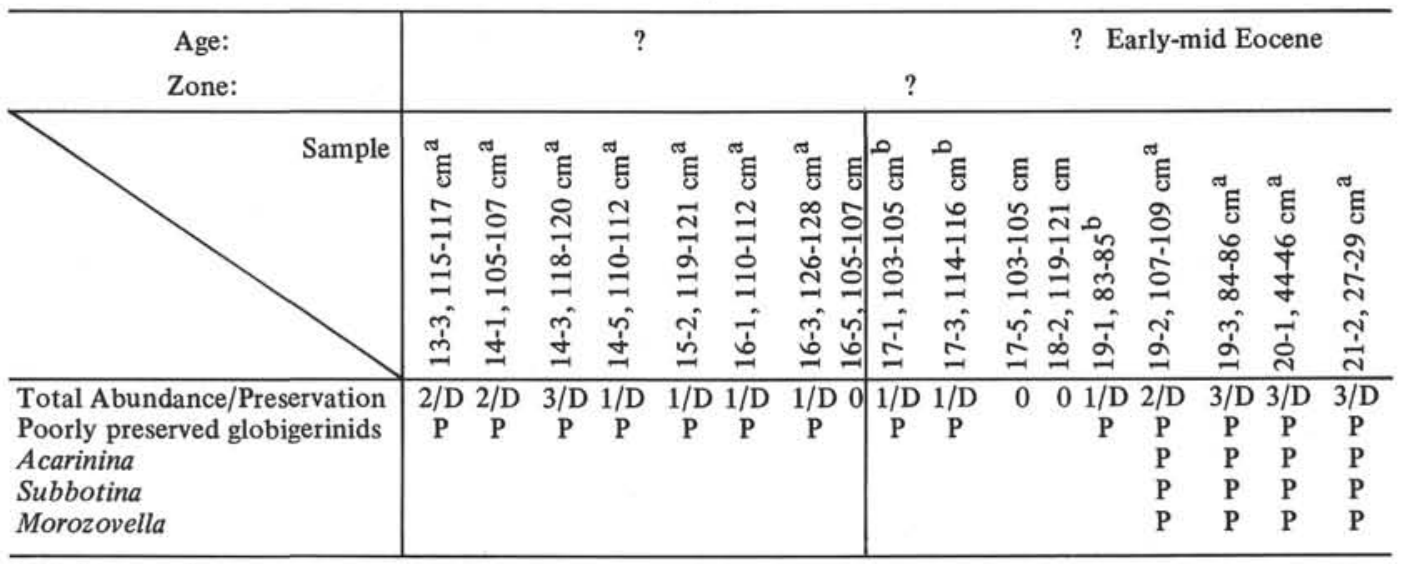

${ }^{\mathrm{a} C h a l k y ~ m a t r i x}$

${ }^{\mathrm{b}}$ Chalky siliceous fossil matrix.

TABLE 12

Distribution of Foraminifera, Hole 207

\begin{tabular}{|c|c|c|c|c|c|c|c|c|c|c|c|c|c|}
\hline Age: & \multicolumn{13}{|c|}{ Pleistocene } \\
\hline \multirow{2}{*}{ ample } & \multicolumn{13}{|c|}{ Globorotalia truncatulinoides } \\
\hline & 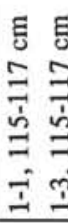 & 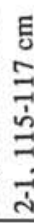 & 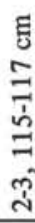 & 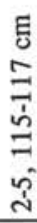 & 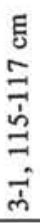 & $\begin{array}{l}E \\
\tilde{\Xi} \\
\Xi \\
\dot{\omega} \\
\Xi \\
\hat{n} \\
\dot{n}\end{array}$ & & $\begin{array}{l}E \\
\Xi \\
\exists \\
\vdots \\
\vdots \\
\dot{J}\end{array}$ & $\begin{array}{l}E \\
\tilde{0} \\
\exists \\
\vec{b} \\
\Rightarrow \\
\dot{g}\end{array}$ & $\begin{array}{l}\Xi \\
0 \\
\Xi \\
\vec{b} \\
\Xi \\
\dot{+}\end{array}$ & $\begin{array}{l}E \\
0 \\
\Xi \\
\vec{b} \\
\exists \\
\vec{n} \\
\vec{n}\end{array}$ & $\begin{array}{l}E \\
\Xi \\
\exists \\
\Xi \\
\text { ம் } \\
\text { ம் }\end{array}$ & 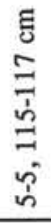 \\
\hline Total Abundance/Preservation & 54 & 4 & 4 & 5 & 4 & 4 & & $4 / \mathrm{D}$ & 4/D & $4 / D$ & 4/D & 4/D & $4 / D$ \\
\hline Globorotalia truncatulinoides & 54 & & 1 & 0 & 0 & 1 & 1 & 0 & 0 & 0 & 0 & 0 & 0 \\
\hline Globorotalia inflata & 54 & 4 & 4 & * & 5 & 5 & 5 & 4 & 5 & 4 & 3 & 5 & 4 \\
\hline Globorotalia crassaformis & 55 & 5 & 5 & * & 5 & 5 & 3 & 4 & 5 & 5 & 4 & 5 & 5 \\
\hline Globorotalia scitula & 22 & 2 & 2 & 2 & 1 & 2 & 2 & 1 & 2 & 2 & 2 & 2 & 2 \\
\hline Orbulina universa & 33 & 3 & 2 & 4 & 3 & 4 & 4 & 4 & 4 & 3 & 5 & 5 & 5 \\
\hline Globigerina quinqueloba & 33 & 3 & 2 & 3 & 2 & 2 & 2 & 2 & 2 & 2 & 2 & 2 & 2 \\
\hline Globigerina bulloides & * 5 & 5 & 5 & * & 5 & 5 & 5 & 5 & 5 & 5 & 5 & 5 & 5 \\
\hline Globigerina pseudopachyderma & * 5 & 5 & 5 & 5 & 5 & 5 & 5 & 5 & 5 & 5 & 5 & 3 & 5 \\
\hline Globigerina falconensis & 55 & 5 & 5 & 4 & 4 & 4 & 4 & 4 & 4 & 4 & 3 & 3 & 3 \\
\hline Globigerina pachyderma & 11 & 1 & 1 & 3 & 1 & 1 & 1 & 1 & 1 & 0 & 0 & 0 & 0 \\
\hline Globigerinella aequilateralis & 11 & 1 & 1 & 0 & 1 & 1 & 1 & 1 & 1 & 1 & 1 & 1 & 1 \\
\hline Globigerinoides rubra & 22 & & 2 & 1 & 1 & 2 & 2 & 1 & 1 & 1 & 1 & 1 & 1 \\
\hline Globigerinoides trilobus & 1 & 1 & 0 & 0 & 0 & 0 & 0 & 0 & 0 & 0 & 1 & 0 & 0 \\
\hline Globigerina dutertrei & & & 0 & 0 & 1 & 1 & 1 & 0 & 0 & 0 & 0 & 0 & 0 \\
\hline Globorotalia hirsuta & & & 1 & 0 & 0 & 0 & 1 & 0 & 0 & 0 & 1 & 1 & 0 \\
\hline Globigerinoides conglobatus & & & 1 & 0 & 0 & 0 & 0 & 0 & 0 & 0 & 0 & 0 & 0 \\
\hline Globigerinita glutinata & 1 & & 3 & 3 & 3 & 3 & 3 & 3 & 2 & 2 & 2 & 2 & 2 \\
\hline
\end{tabular}


TABLE 13

Distribution of Foraminifera, Hole 207A

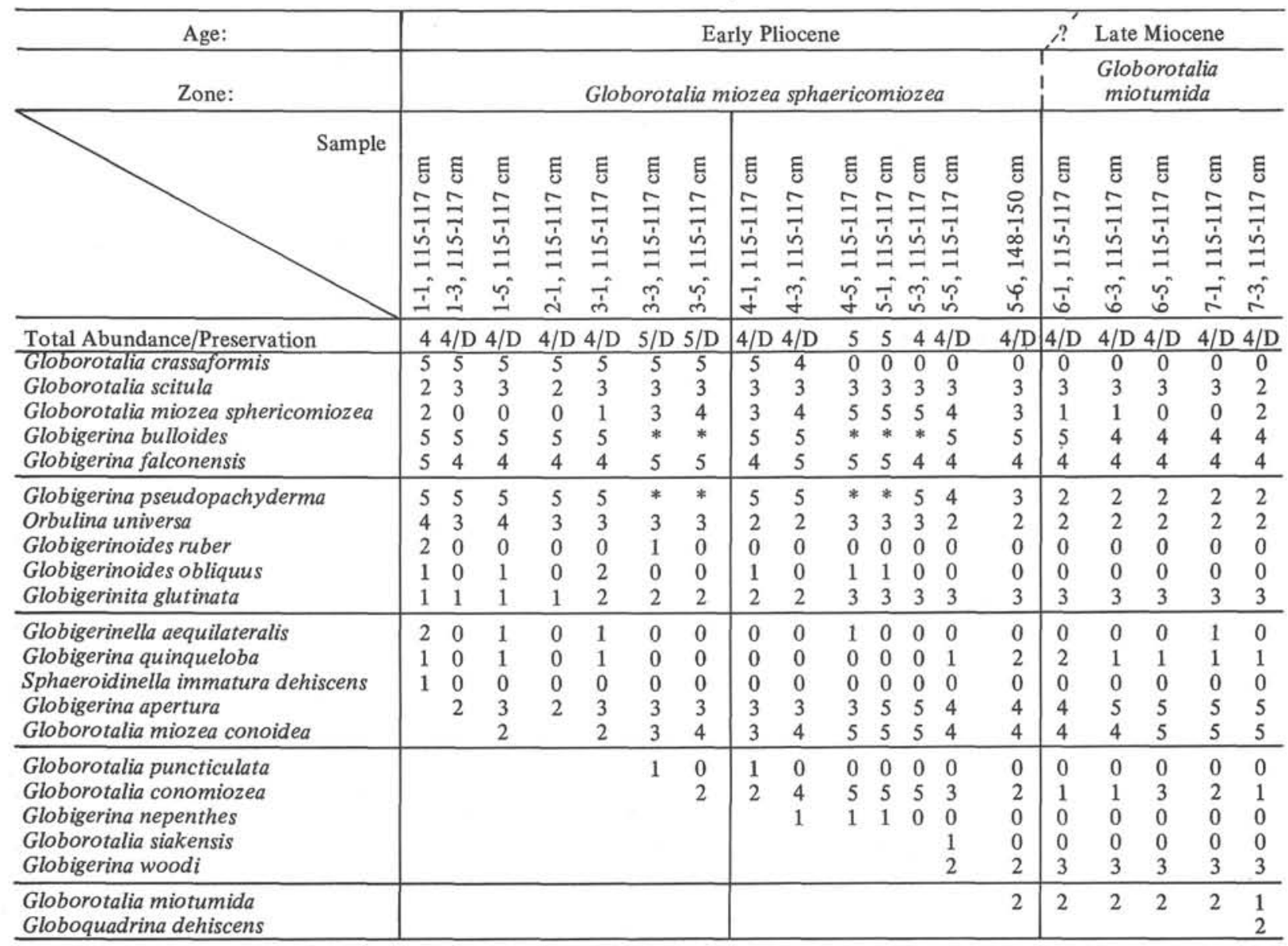


TABLE 14

Distribution of Foraminifera, Hole 207A - Continued

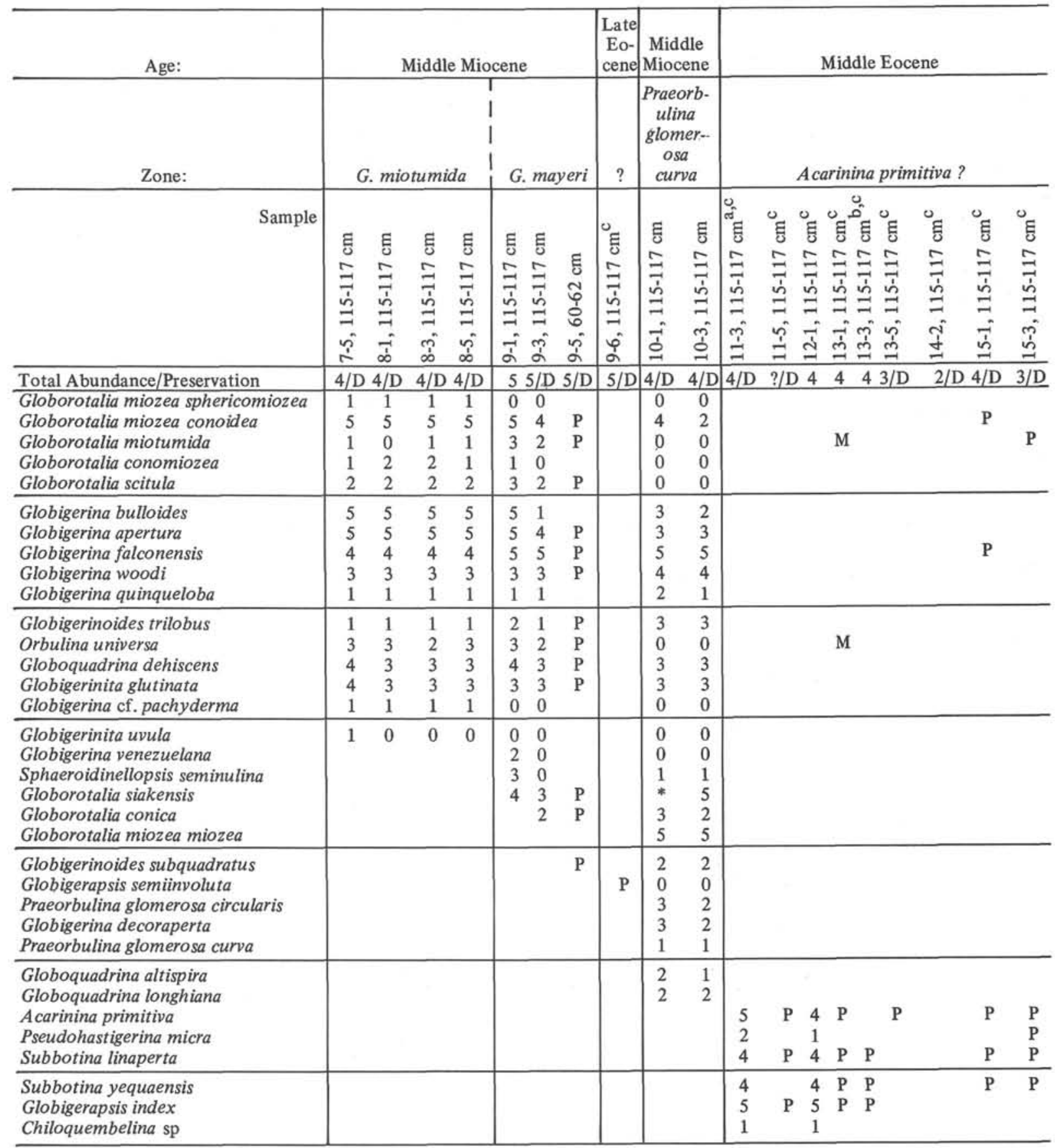

${ }^{\mathrm{a}}$ Others difficult to identify.

${ }^{b}$ Like Eocene above but with Miocene-Pleistocene species.

${ }^{\mathrm{c}}$ Chalky matrix. 
TABLE 15

Distribution of Foraminifera, Hole 207A - Continued

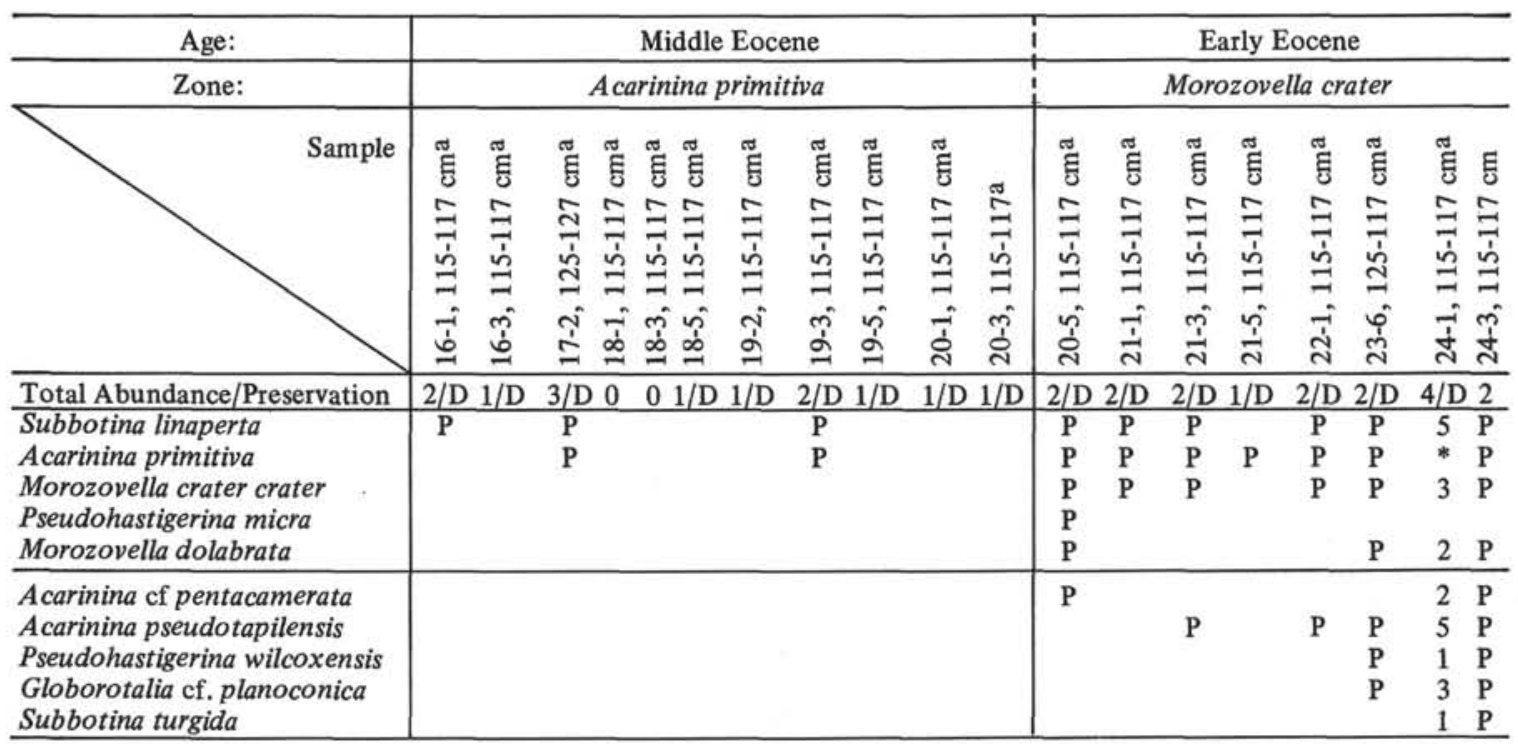

${ }^{\mathrm{a}}$ Chalk

TABLE 16

Distribution of Foraminifera, Hole 207A - Continued

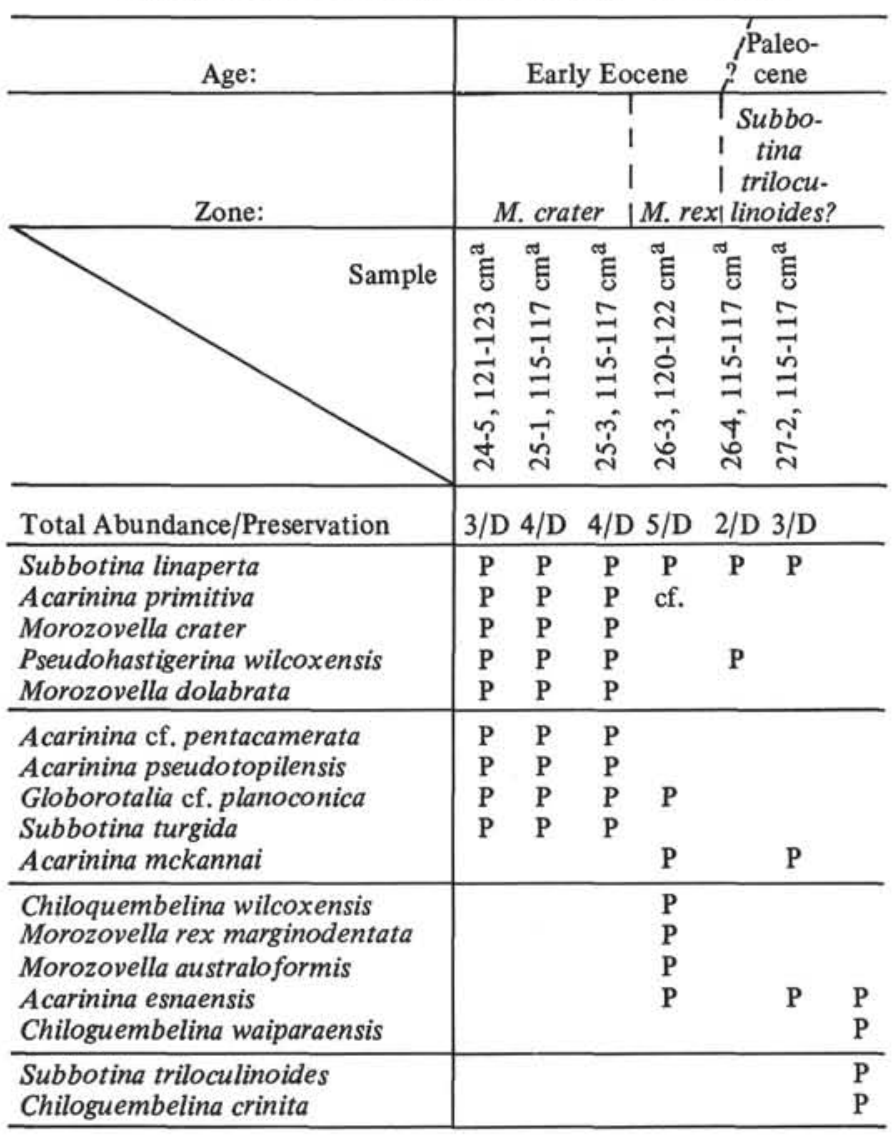

${ }^{\text {a }}$ Chalk 
TABLE 17

Distribution of Foraminifera, Hole 208

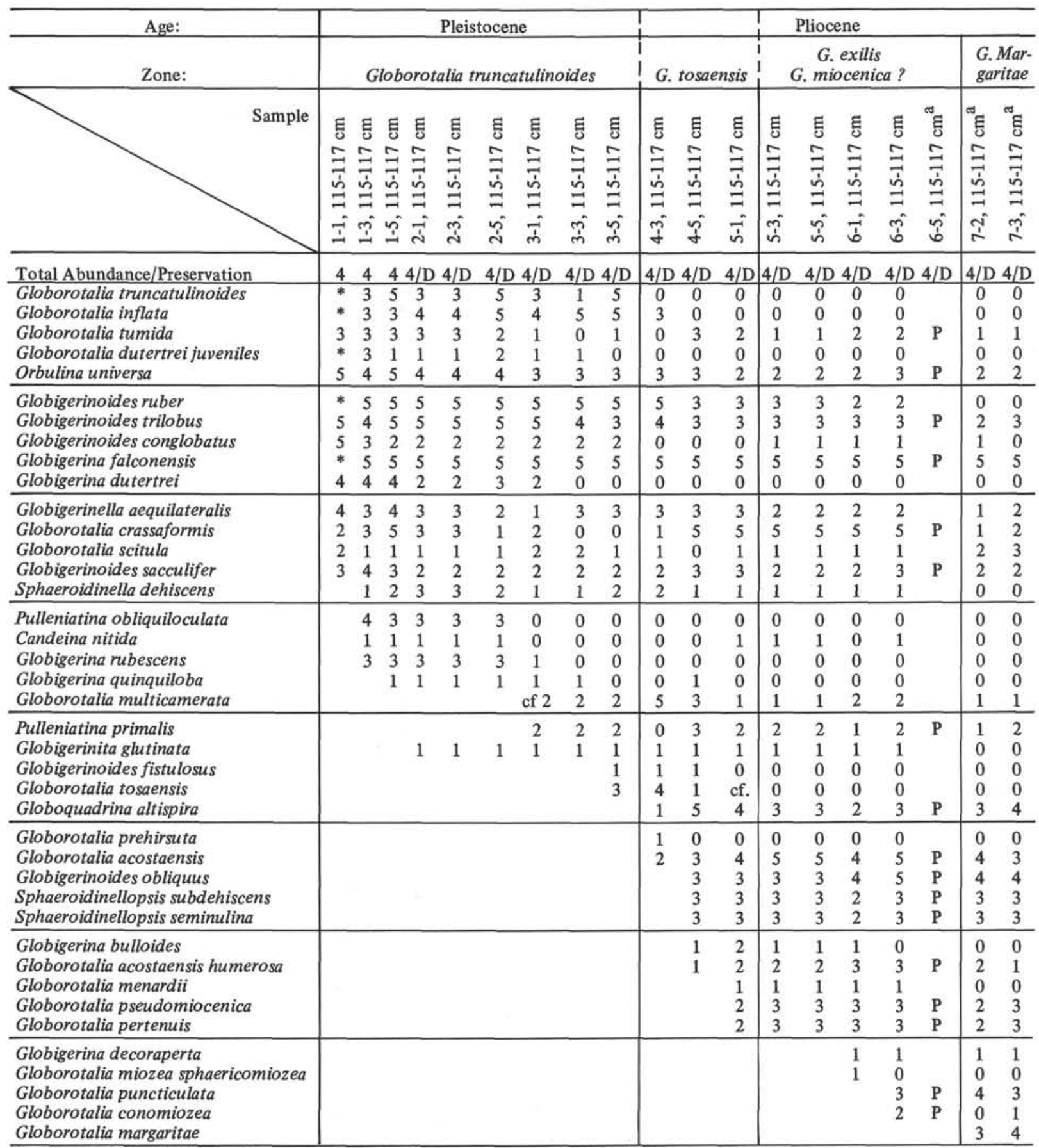

${ }^{\mathrm{a}}$ Chalk 
TABLE 18

Distribution of Foraminifera, Hole 208 - Continued

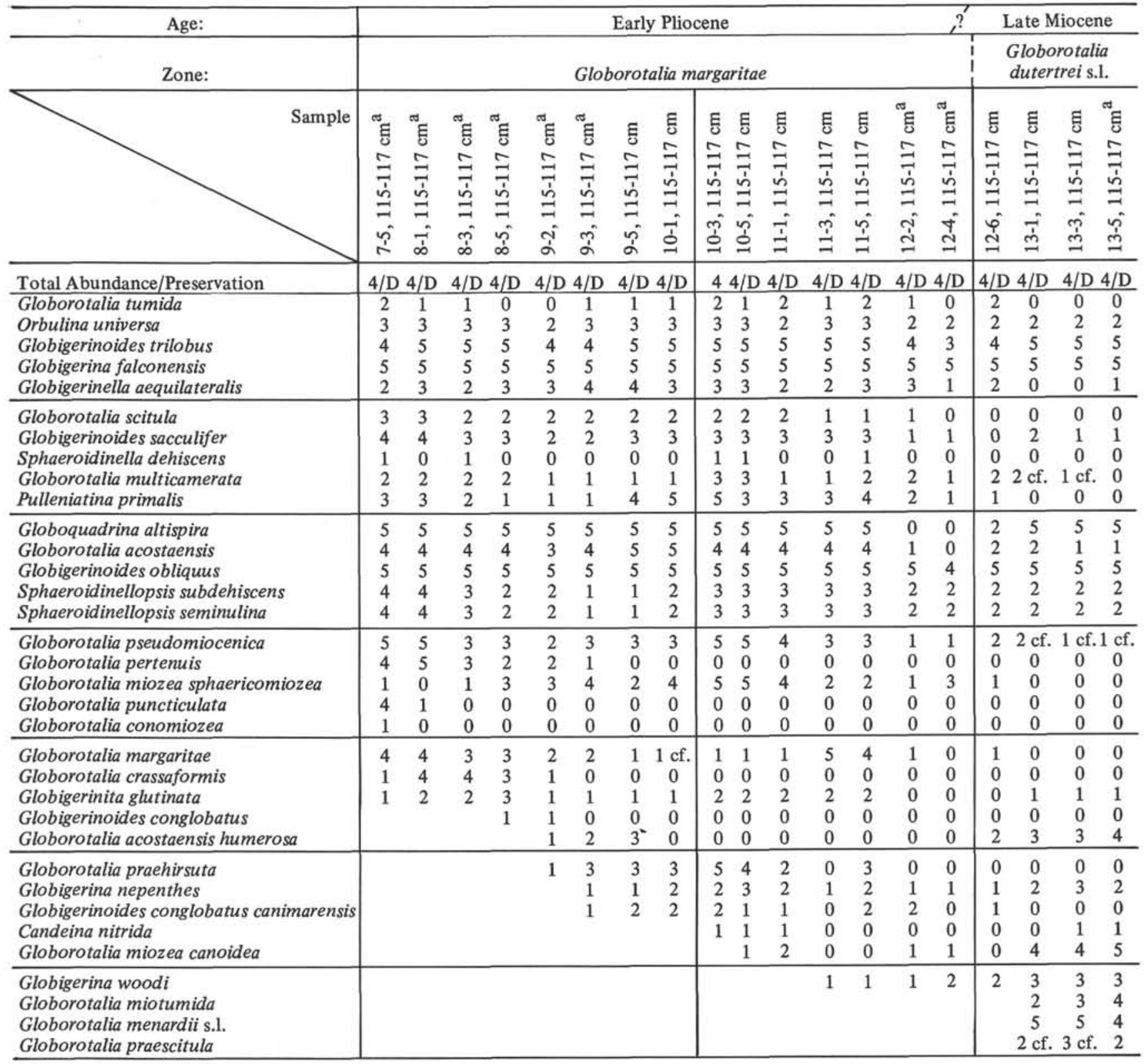

${ }^{\mathrm{a}}$ Chalk 
TABLE 19

Distribution of Foraminifera, Hole 208 - Continued

\begin{tabular}{|c|c|c|c|c|c|c|c|c|c|c|c|c|c|c|c|c|c|c|c|}
\hline Age: & \multicolumn{13}{|c|}{ Late Miocene } & \multicolumn{6}{|c|}{ Miocene } \\
\hline Zone: & \multicolumn{13}{|c|}{ Globorotalia plesiotumida } & \multicolumn{6}{|c|}{$?$} \\
\hline Sample & 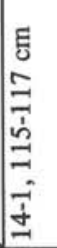 & 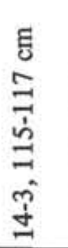 & 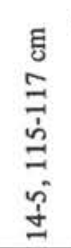 & 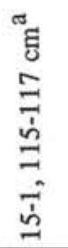 & 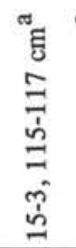 & 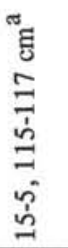 & 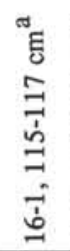 & 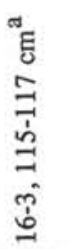 & 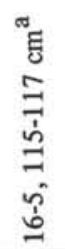 & 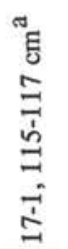 & 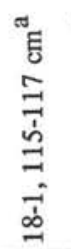 & 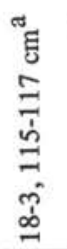 & 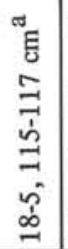 & 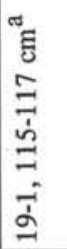 & 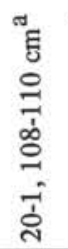 & 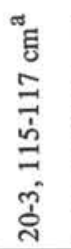 & 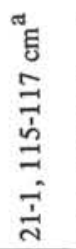 & 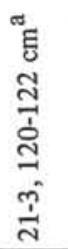 & 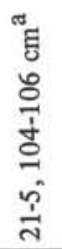 \\
\hline Total Abundance/Preservation & $4 / \mathrm{D}$ & $4 / D$ & $4 / D$ & $4 / D$ & $4 / D$ & $4 / D$ & $4 / D$ & 4/D & $3 / \mathrm{D}$ & $4 / D$ & 4/D & $3 / \mathrm{D}$ & $4 / D$ & $3 / \mathrm{D}$ & $3 / \mathrm{D}$ & $3 / \mathrm{D}$ & $2 / \mathrm{D}$ & $3 / \mathrm{D}$ & $2 / \mathrm{D}$ \\
\hline $\begin{array}{l}\text { Orbulina universa and } O . \\
\quad \text { suturalis } \\
\text { Globigerinoides trilobus } \\
\text { Globigerina falconensis } \\
\text { Globigerinella aequilateralis } \\
\text { Globigerinoides sacculifer } \\
\end{array}$ & $\begin{array}{l}2 \\
5 \\
5 \\
1 \\
2 \\
\end{array}$ & $\begin{array}{l}2 \\
5 \\
5 \\
1 \\
1\end{array}$ & $\begin{array}{l}2 \\
5 \\
5 \\
1 \\
1\end{array}$ & $\begin{array}{l}\mathbf{P} \\
\mathbf{P} \\
\mathbf{P}\end{array}$ & $\begin{array}{l}P \\
P\end{array}$ & $\begin{array}{l}\mathbf{P} \\
\mathbf{P}\end{array}$ & $\begin{array}{l}\mathbf{P} \\
\mathbf{P} \\
\mathbf{P}\end{array}$ & $\begin{array}{l}P \\
P\end{array}$ & $\mathbf{P}$ & $\mathbf{P}$ & $\mathbf{P}$ & $P$ & $P$ & $\mathbf{P}$ & & & & $P$ & \\
\hline $\begin{array}{l}\text { Globoquadrina altispira } \\
\text { Globorotalia acostaensis } \\
\text { Globigerinoides obliquus } \\
\text { Sphaeroidinellopsis seminulina } \\
\text { Sphaeroidinellopsis subdehiscens }\end{array}$ & $\begin{array}{l}5 \\
2 \\
5 \\
2 \\
2\end{array}$ & $\begin{array}{l}5 \\
1 \\
5 \\
2 \\
2\end{array}$ & $\begin{array}{l}5 \\
2 \\
5 \\
2 \\
2\end{array}$ & $\begin{array}{l}\mathrm{P} \\
\mathrm{P} \\
\mathrm{P} \\
\mathrm{P}\end{array}$ & $\begin{array}{l}\mathrm{P} \\
\mathrm{P} \\
\mathrm{P} \\
\mathrm{P} \\
\mathrm{P}\end{array}$ & $\begin{array}{l}\mathbf{P} \\
\mathbf{P} \\
\mathbf{P} \\
\mathbf{P}\end{array}$ & $\begin{array}{l}\mathbf{P} \\
\mathrm{P} \\
\mathrm{P} \\
\mathrm{P} \\
\mathrm{P}\end{array}$ & $\begin{array}{l}\mathrm{P} \\
\mathrm{P} \\
\mathrm{P} \\
\mathrm{P}\end{array}$ & $P$ & $\begin{array}{l}\mathrm{P} \\
\mathrm{P}\end{array}$ & $\begin{array}{l}\mathrm{P} \\
\mathrm{P}\end{array}$ & $\begin{array}{l}\mathrm{P} \\
\mathrm{P}\end{array}$ & $\begin{array}{l}\mathrm{P} \\
\mathrm{P} \\
\mathrm{P} \\
\mathrm{P}\end{array}$ & & & & & $\mathbf{P}$ & $\mathrm{P}$ \\
\hline $\begin{array}{l}\text { Globigerina nepenthes } \\
\text { Condeina nitida } \\
\text { Globigerina woodi } \\
\text { Globorotalia humerosa } \\
\text { Globorotalia menardii s.l. } \\
\end{array}$ & $\begin{array}{l}2 \\
1 \\
1 \\
2 \\
4\end{array}$ & $\begin{array}{l}2 \\
0 \\
1 \\
3 \\
3 \\
\end{array}$ & $\begin{array}{l}1 \\
0 \\
1 \\
2 \\
2 \\
\end{array}$ & $\mathbf{P}$ & $\mathbf{P}$ & & $\begin{array}{l}\mathrm{P} \\
\mathrm{P} \\
\mathrm{P}\end{array}$ & & $\mathbf{P}$ & $\mathbf{P}$ & $\mathbf{P}$ & $\mathbf{P}$ & $\mathbf{P}$ & $\mathbf{P}$ & $\mathrm{P}$ & & & & \\
\hline $\begin{array}{l}\text { Globorotalia praescitula } \\
\text { Globorotalia plesiotumida } \\
\text { Globorotalia merotumida } \\
\text { Globorotalia miotumida } \\
\text { Globigerinita glutinata }\end{array}$ & $\begin{array}{l}3 \\
3 \\
2 \\
1 \\
1\end{array}$ & $\begin{array}{l}3 \\
3 \\
2 \\
1 \\
1\end{array}$ & $\begin{array}{l}3 \\
1 \\
2 \\
4 \\
1\end{array}$ & $\begin{array}{l}\mathrm{P} \\
\mathrm{P} \\
\mathrm{P} \\
\mathrm{P}\end{array}$ & $\begin{array}{l}P \\
\text { cf. } \\
P\end{array}$ & $\begin{array}{l}\mathrm{P} \\
\mathrm{P} \\
\mathrm{P} \\
\mathrm{P}\end{array}$ & $\begin{array}{l}P \\
P \\
P \\
P \\
P\end{array}$ & $\begin{array}{l}P \\
P\end{array}$ & $\begin{array}{l}\mathbf{P} \\
\mathbf{P}\end{array}$ & $\begin{array}{l}\mathbf{P} \\
\mathbf{P}\end{array}$ & $\begin{array}{l}\mathbf{P} \\
\mathbf{P}\end{array}$ & $\begin{array}{l}\mathbf{P} \\
\mathbf{P}\end{array}$ & $\begin{array}{l}P \\
P \\
P\end{array}$ & & & & & & \\
\hline $\begin{array}{l}\text { Globorotalia obesa } \\
\text { Globorotalia miozea conoidea } \\
\text { Globorotalia miozea miozea } \\
\text { Globoquadrina dehiscens }\end{array}$ & & & 1 & $\mathbf{P}$ & $\mathbf{P}$ & $P$ & $P$ & $\mathbf{P}$ & $\mathbf{P}$ & $\begin{array}{l}P \\
P\end{array}$ & $\begin{array}{l}P \\
P\end{array}$ & $\mathbf{P}$ & $\mathbf{P}$ & $\begin{array}{l}P \\
P\end{array}$ & $\begin{array}{l}P \\
P\end{array}$ & $\begin{array}{c}\mathrm{P} \\
\text { cf. } \\
\mathrm{P}\end{array}$ & $P$ & $P$ & $\mathbf{P}$ \\
\hline
\end{tabular}

${ }^{\mathrm{a}}$ Chalk

TABLE 20

Distribution of Foraminifera, Hole 208 - Continued

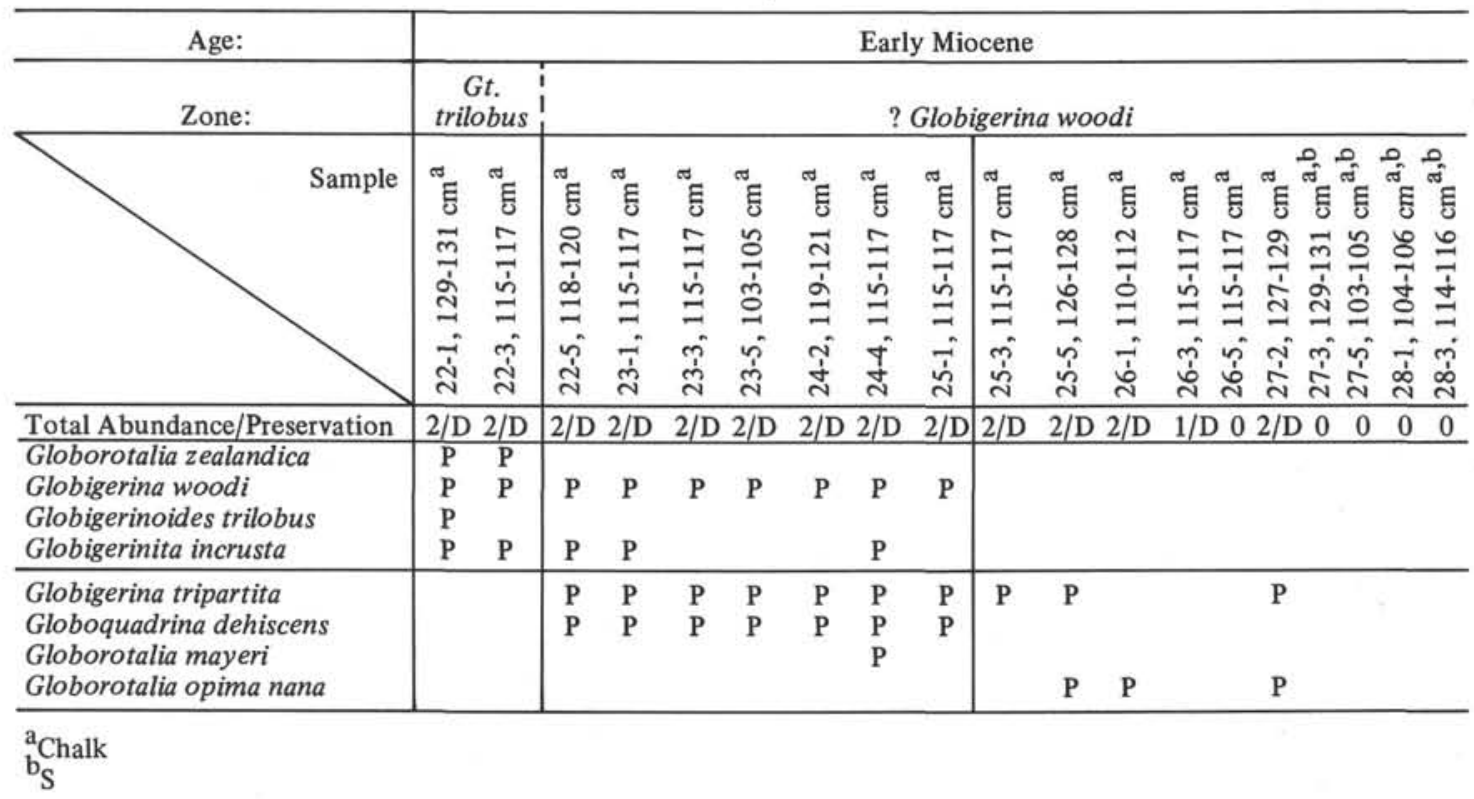


TABLE 21

Distribution of Foraminifera, Hole 208 - Continued

\begin{tabular}{|c|c|}
\hline Age: & $?$ \\
\hline Zone: & $?$ \\
\hline Sample & 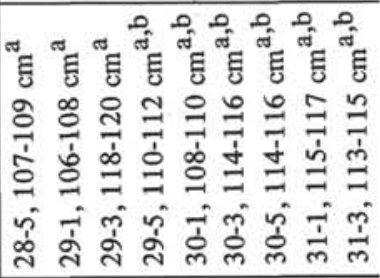 \\
\hline Total Abundance/Preservation & 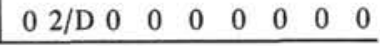 \\
\hline
\end{tabular}

TABLE 22

Distribution of Foraminifera, Hole 209

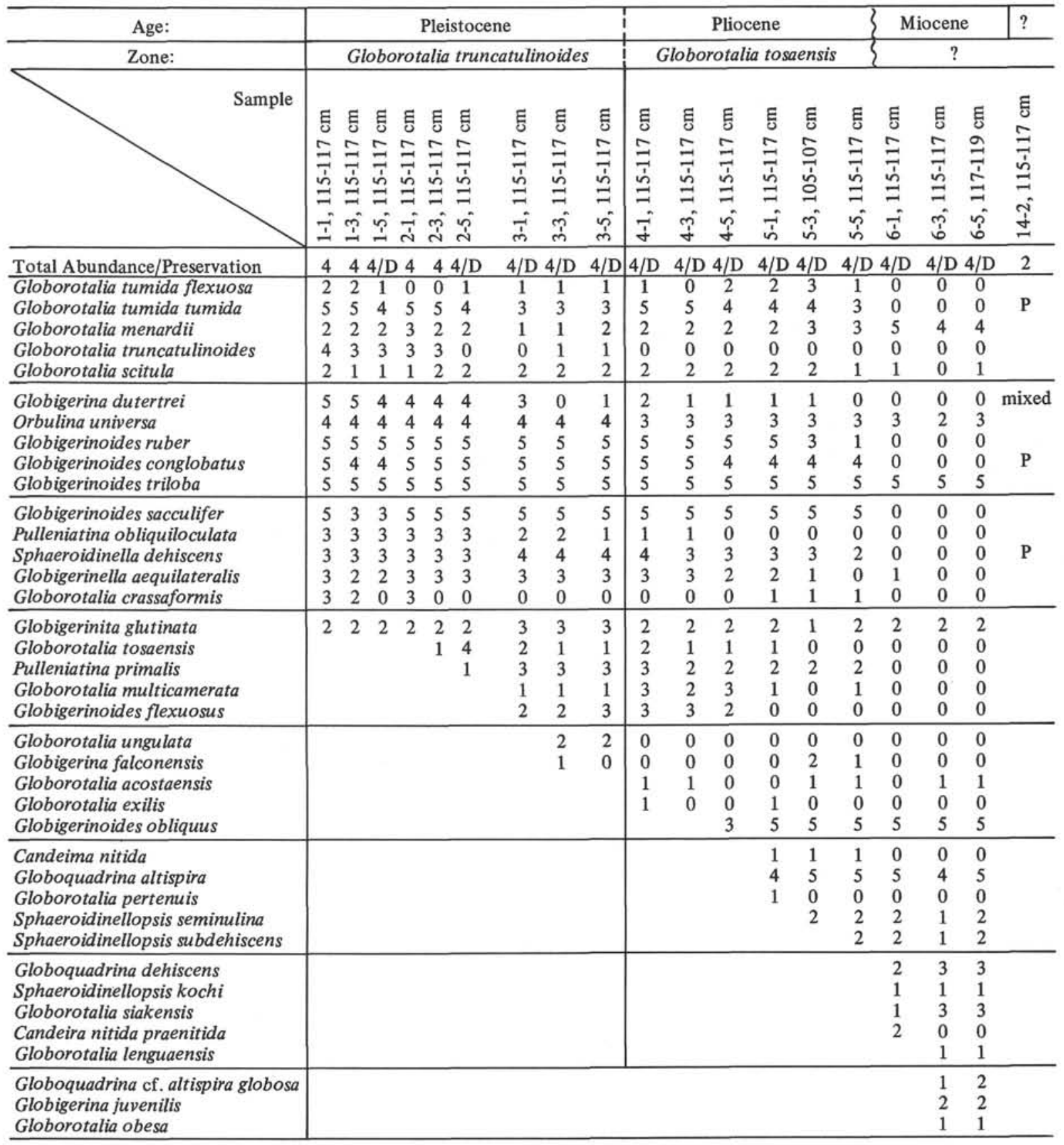


TABLE 23

Distribution of Foraminifera, Hole 209 - Continued

\begin{tabular}{|c|c|c|c|c|c|c|c|c|c|c|c|c|}
\hline Age: & \multicolumn{2}{|r|}{$?$} & \multicolumn{4}{|c|}{$\begin{array}{c}\text { Late } \\
\text { Eocene }\end{array}-\begin{array}{c}\text { Early } \\
\text { Oligo. }\end{array}$} & \multicolumn{6}{|c|}{ Middle Eocene } \\
\hline Zone: & \multicolumn{2}{|r|}{$?$} & \multicolumn{4}{|c|}{$?$} & \multicolumn{6}{|c|}{ ? } \\
\hline Sample & 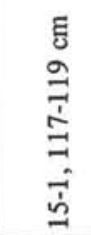 & 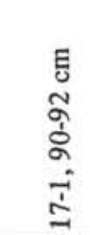 & 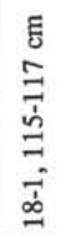 & 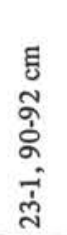 & 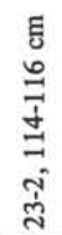 & $\begin{array}{l}\text { Ė } \\
\infty \\
\frac{1}{1} \\
\underline{-1} \\
\vec{j}\end{array}$ & 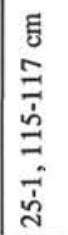 & 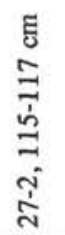 & 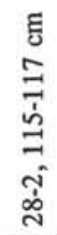 & 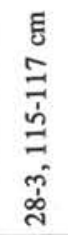 & 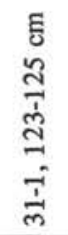 & 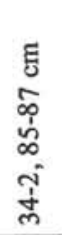 \\
\hline Total Abundance/Preservation & 2 & 1 & $3 / \mathrm{D}$ & 2/D & $2 / \mathrm{D}$ & 3/D & 3/D & $4 / \mathrm{D}$ & $3 / \mathrm{D}$ & $3 / \mathrm{D}$ & 2/D & $1 / \mathrm{D}$ \\
\hline $\begin{array}{l}\text { Globigerinoides ruber } \\
\text { Globigerinoides sacculifer } \\
\text { Globigerinoides conglobatus } \\
\text { Pseudohastigerina micra } \\
\text { Chiloguembelina cubensis } \\
\end{array}$ & $\begin{array}{l}\text { mixed } \\
\text { mixed }\end{array}$ & mixed & $\begin{array}{l}2 \\
1\end{array}$ & $\begin{array}{l}1 \\
1\end{array}$ & 1 & $\begin{array}{l}1 \\
1\end{array}$ & $\begin{array}{l}0 \\
0\end{array}$ & $\begin{array}{l}0 \\
0\end{array}$ & & & & \\
\hline $\begin{array}{l}\text { Globigerina praebulloides } \\
\text { Globigerinita martini } \\
\text { Globigerina tapuriensis } \\
\text { Globigerapsis index } \\
\text { Globigerina angustiumbilicata } \\
\end{array}$ & & & $\begin{array}{l}5 \\
1\end{array}$ & $\begin{array}{l}3 \\
1 \\
2 \\
?\end{array}$ & $\begin{array}{l}1 \\
1 \\
1 \\
?\end{array}$ & $\begin{array}{l}3 \\
2 \\
1 \\
2 \\
1\end{array}$ & $\begin{array}{l}0 \\
0 \\
0 \\
3 \\
0 \\
\end{array}$ & $\begin{array}{l}0 \\
0 \\
0 \\
5 \\
0 \\
\end{array}$ & $\mathbf{P}$ & $\mathbf{P}$ & & $\mathbf{P}$ \\
\hline $\begin{array}{l}\text { Globigerina unicava } \\
\text { Subbotina angiporoides } \\
\text { Truncorotaloides collactea } \\
\text { Globorotalia cerroazulensis cerroazulensis } \\
\text { Truncorotaloides pseudotopilensis }\end{array}$ & & & & & & $\begin{array}{l}2 \\
1\end{array}$ & $\begin{array}{l}0 \\
0 \\
3 \\
1 \\
2\end{array}$ & $\begin{array}{l}0 \\
0 \\
5 \\
4 \\
5\end{array}$ & $\mathbf{P}$ & $\begin{array}{l}\mathrm{P} \\
\mathrm{P}\end{array}$ & $\mathrm{P}$ & \\
\hline $\begin{array}{l}\text { Subbotina linaperta } \\
\text { Morozovella spinulosa } \\
\text { Acarinina primitiva } \\
\text { Subbotina yequaensis } \\
\text { Acarinina densa } \\
\text { "Globorotalia" cerroazulensis pomeroli }\end{array}$ & & & & & & & 1 & $\begin{array}{l}2 \\
2 \\
3 \\
5\end{array}$ & $\begin{array}{l}\mathrm{P} \\
\mathrm{P} \\
\mathrm{P} \\
\mathrm{P} \\
\mathrm{P}\end{array}$ & $\begin{array}{l}P \\
P \\
P \\
P \\
P \\
P\end{array}$ & $\begin{array}{l}\mathrm{P} \\
\mathrm{P}\end{array}$ & \\
\hline
\end{tabular}

TABLE 24

Distribution of Foraminifera, Hole 210

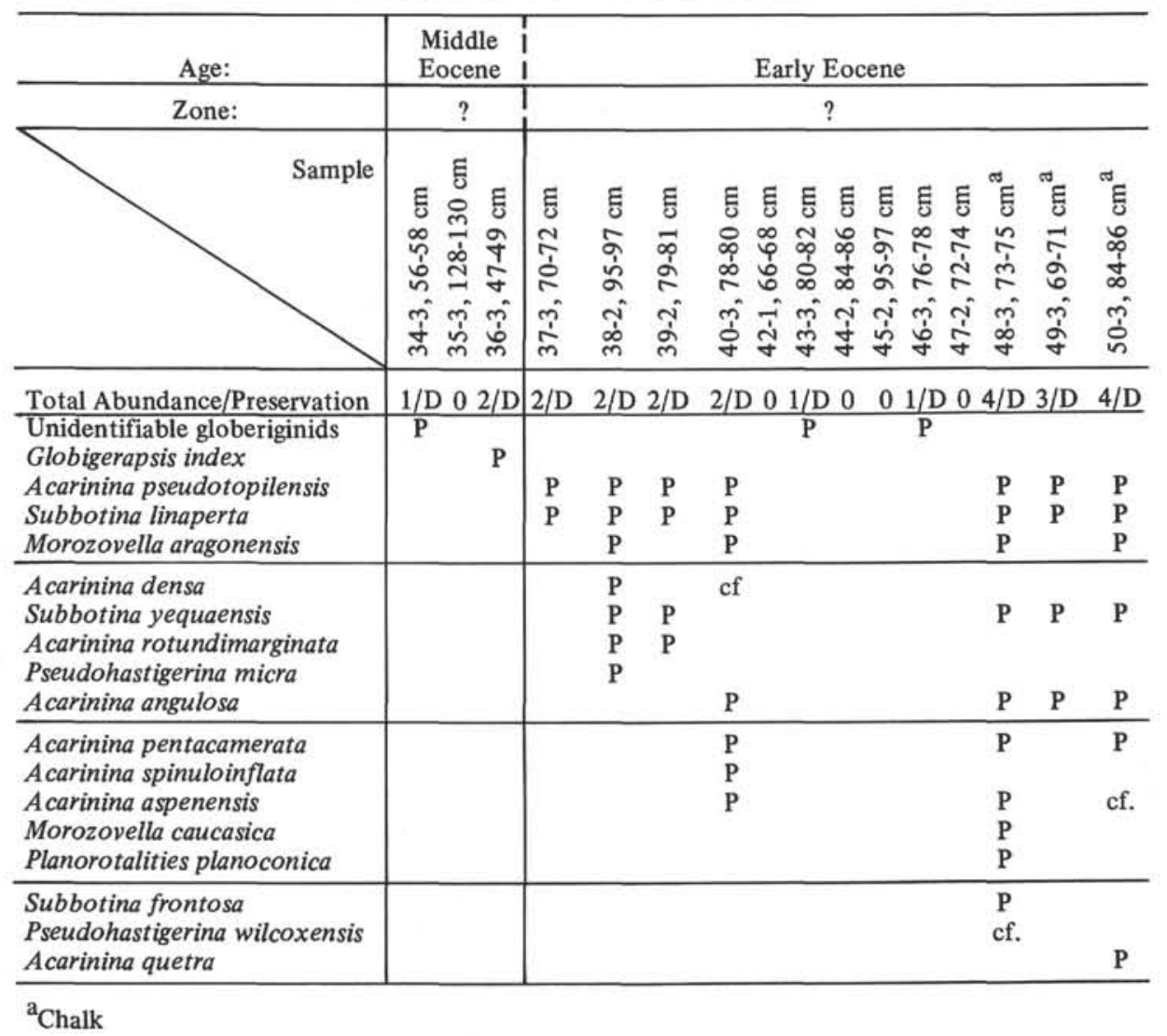

